\title{
Implementation of a Multicenter Performance Improvement Program for Early Detection and Treatment of Severe Sepsis in General Medical-Surgical Wards
}

\author{
Christa Schorr, RN, MSN, Andrew Odden, MD, SFHM², Laura Evans, MD, MSc ${ }^{3}$, Gabriel J. Escobar, MD4, \\ Snehal Gandhi, MD${ }^{5}$, Sean Townsend, MD6, Mitchell Levy, MD
}

\begin{abstract}
${ }^{1}$ Cooper Research Institute-Critical Care, Cooper University Hospital, Camden, New Jersey; ${ }^{2}$ Department of Medicine, Washington University School of Medicine, Saint Louis, Missouri; ${ }^{3}$ Division of Pulmonary, Critical Care, and Sleep Medicine, New York University School of Medicine, New York, New York: ${ }^{4}$ Systems Research Initiative, Kaiser Permanente Division of Research, Kaiser Permanente, Oakland, California; ${ }^{5}$ Division of Hospital Medicine, Medical Informatics and Care Delivery Innovation, Cooper University Hospital, Camden, New Jersey; ${ }^{6}$ Department of Quality and Safety, California Pacific Medical Center, San Francisco, California; ${ }^{7}$ Department of Pulmonary, Critical Care, and Sleep Medicine, Rhode Island Hospital, Providence, Rhode Island.
\end{abstract}

\begin{abstract}
Sepsis is a leading cause of in-hospital death, and evidence suggests a higher mortality in patients presenting with sepsis on the ward compared to those presenting to the emergency department. Ward patients who develop severe sepsis may have poor outcomes for a variety of reasons, including delayed diagnosis, lack of readily available staffing, and delayed treatment. We report on a multihospital quality improvement program for early detection and treatment of sepsis on general medical-surgical wards. We describe a multipronged approach to improve severe sepsis outcomes using the Institute for Healthcare Improvement's Plan-Do-Study-Act model. Sixty sites engaged in a collaborative implementation process that aligned people, process, and technology. Based on our experience, we recommend
\end{abstract}

a stepwise approach to implement such a program: (1) both administrative and clinical leadership commit to a common goal; (2) appoint clinical champions and give them authority to engage other clinicians to improve timeliness of interventions; (3) map workflows and processes to rely heavily on the nursing staff's ability to evaluate and report severe sepsis screening results; (4) if available, design and deploy technology with the assistance of clinical informaticians (eg, to enable electronic health records-based continuous screening); (5) to determine success, consider tracking screening compliance and process, and outcome measures such as length of stay and mortality. Journal of Hospital Medicine 2016;S11:32-S39. (c) 2016 Society of Hospital Medicine
Sepsis, the body's systemic response to infection leading to organ failure, can occur in patients throughout the hospital. However, patients initially diagnosed with sepsis on the wards experience the highest mortality for several reasons, including delayed recognition and treatment, particularly when localized infections progress to shock and organ failure. Consequently, hospitals have responded by having nurses screen patients for signs and symptoms of sepsis to identify cases earlier and improve outcomes. The intent of this article, which is based on our experience with a multihospital implementation effort, was to describe potential reasons for ward patients' poor prognosis. We provide a toolkit for how hospitals can implement a severe sepsis quality improvement $(\mathrm{QI})$ program in general medical-surgical wards.

In a previous study, we reported on our international effort, the Surviving Sepsis Campaign's (SSC) Phase III

\footnotetext{
*Address for correspondence and reprint requests: Christa Schorr, Cooper Research Institute-Critical Care, Cooper University Hospital, One Cooper Plaza, Dorrance Building, Suite 411, Camden, NJ 08103; Telephone: 856-968-7493; Fax: 856-968-8378;

E-mail: schorr-christa@cooperhealth.edu
}

Additional Supporting Information may be found in the online version of this article.

Received: March 12, 2016; Revised: August 26, 2016; Accepted: August 31, 2016

2016 Society of Hospital Medicine DOI 10.1002/jhm.2656

Published online in Wiley Online Library (Wileyonlinelibrary.com). performance improvement (PI) program, targeting selected guideline recommendations (6- and 24-hour bundles) in the emergency department (ED), the Intensive Care Unit (ICU), and wards in 165 volunteer hospitals in the United States, Europe, and South America. ${ }^{1}$ The program was associated with increased bundle compliance and decreased mortality over time. ${ }^{1,2}$ The SSC's Phase III program, which focused on improvement efforts primarily in the ED and ICU, also exposed a need to address the high mortality in ward patients. ${ }^{3}$ Patients admitted to the ICU directly from the ED with severe sepsis had a mortality rate of $26 \%$, whereas those transferred to the ICU from the ward had significantly higher mortality $(40.3 \%) .^{3}$

Although the reasons for the higher mortality rate among ward patients have not been studied, several factors may play a role. First, the diagnosis of severe sepsis may be delayed in ward patients because physicians and nurses may not recognize the progression to sepsis and/or because hospitalized patients may not present with obvious systemic manifestations of sepsis as they do in the ED (Table 1). ${ }^{4}$ Second, ward patients may have differences in the timing of their presentation and concurrent conditions confounding the diagnosis. ${ }^{5}$ Third, treatment may be delayed once the diagnosis is made on the ward. The ICU and ED are designed to provide rapid highacuity care, whereas the wards have fewer systems and resources for rapid delivery of care needed for severe 
TABLE 1. Presentation of Severe Sepsis in the Emergency Department and the Ward

\begin{tabular}{|c|c|c|}
\hline & Emergency Department Presentation & Ward Presentation \\
\hline Patient-family-reported symptoms & "l just feel sick," "family reports disorientation," "not eating" & $\begin{array}{l}\text { Currently hospitalized, family often not present, diagnosis may not be clear, } \\
\text { baseline mental status unknown, lack of appetite may be linked to } \\
\text { dislike of hospital food. }\end{array}$ \\
\hline Systemic manifestations & $\begin{array}{l}\text { Triage observed } 2 \text { or more signs of infection or patient reports } \\
\text { temperature while at home plus additional finding on assessment. }\end{array}$ & $\begin{array}{l}\text { Signs of infection may appear } 1 \text { at a time, hours apart, } \\
\text { and may appear to be mild changes to staff } \\
\text { or missed entirely due to staff discontinuity. }\end{array}$ \\
\hline Organ dysfunction & Present on admission; triage nurse assesses for organ dysfunction. & Develops over hours or days; may be subtle or acute. \\
\hline Laboratory study process & Ordered and evaluated within 1 hour. & $\begin{array}{l}\text { Not routinely completed daily, may be ordered after physician } \\
\text { evaluation or during rounds. Results within 3-4 hours. }\end{array}$ \\
\hline
\end{tabular}

sepsis. Finally, some patients on the ward may develop sepsis from nosocomial infection, which can portend a worse prognosis. ${ }^{6}$

The SSC Phase III results led to the launch of a QI program, known as the SSC Phase IV Sepsis on the Wards Collaborative, funded by the Gordon and Betty Moore Foundation. This program, a partnership between the Society of Critical Care Medicine and the Society of Hospital Medicine (SHM), targeted ward patients and focused on early recognition through protocol-driven regular nurse screening. The program applied the SSC 2012 guidelines with a primary focus on the 3-hour bundle (Table 2). ${ }^{7}$ The framework used for this program was the Institute for Healthcare Improvement's Plan-Do-Study-Act (PDSA) model of improvement. 8 The collaborative design included learning sessions designed to motivate and support improvement. ${ }^{10}$ The program began with 60 academic and community hospitals in 4 US regions. Participating sites were required to have prior hospital experience in sepsis performance improvement as well as a formal commitment of support from their EDs and ICUs.

We provided sites with a basic screening tool and guidance for routine severe sepsis screening, monitoring, and feedback (Figure 1). Because of the anticipated challenges of implementing routine nurse screening on every shift in all inpatient wards, participants identified 1 ward to pilot the every-shift screening program. Each pilot ward refined the nurse screening process and developed site-specific tools based on electronic health record (EHR) capability, informatics support, and available resources. After this initial phase, the program could be implemented in a hospital's remaining wards. The slogan adopted for the program was "Screen every patient, every shift, every day."

TABLE 2. Surviving Sepsis Campaign 3-Hour Severe Sepsis Bundle

To be completed within 3 hours of time of presentation

1. Measure lactate level

2. Obtain blood cultures prior to administration of antibiotics

3. Administer broad-spectrum antibiotics

4. Administer $30 \mathrm{~mL} / \mathrm{kg}$ crystalloid for hypotension or lactate $\geq 4 \mathrm{mmol} / \mathrm{L}$ ( $36 \mathrm{mg} / \mathrm{dL}$ )
Although knowledge gained from the SSC Phase III program led to improvements in treating severe sepsis, ward patients continued to have poor outcomes. To address the potential contributions of delayed case identification, we developed an early recognition and treatment program. We outline the steps we took to develop this multisite PI program.

\section{PREPARATORY WORK}

During the planning phase, several procedural steps were taken before initiating the ward sepsis program (Table 3). These required 3 levels of involvement: senior administration, midlevel management, and patient-level support.

\section{Administrative Support}

In the course of our implementation effort, we found that sites that had high-level administrative support were more likely to implement and sustain the intervention. For this reason, we consider such support to be critical. Examples of such support include chief medical officers, chief nursing officers, and chief quality officers. As an example, securing commitment from hospital leadership may be necessary to improve/ change the EHR and provide funding for project management to achieve sustainable improvement in outcomes. Aligning leadership with frontline physicians, nurses, and support staff toward a common goal provides the platform for a successful program. ${ }^{11}$

\section{ED and ICU Leadership Support}

Maintaining lines of communication among the ED, ICU, and ward staff is critical to improving outcomes. Establishing a cohesive system (ED, ICU, and wards) aimed at early recognition and treatment of sepsis throughout the hospital stay can lead to improvement in continuity of care and outcomes. For example, when an ED severe sepsis patient is transferred to the ward and subsequently requires admission to the ICU due to declining clinical status, providing timely feedback to the ED can help improve care for subsequent patients. Collaboration between the $\mathrm{ED}$ and the ward can also contribute to improved transitions of care for patients with severe sepsis.

\section{Hospitalist/Internal Medicine Leadership}

Our experience with implementing sepsis bundles in the ED and ICU highlights the need for effective interdisciplinary collaboration with designated physician 


\section{Evaluation for Severe Sepsis Screening Tool}

\section{Instructions: Use this optional tool to screen patients for severe sepsis in the emergency department, on} the medical/surgical floors, or in the ICU.

1. Is the patient's history suggestive of a new infection?

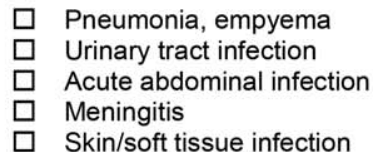

$\begin{array}{ll}\square & \text { Bone/joint infection } \\ \square & \text { Wound infection } \\ \square & \text { Blood stream catheter } \\ \text { infection } \\ \square \quad \text { Endocarditis }\end{array}$

Implantable device infection

Other infection

Skin/soft tissue infection

Endocarditis

2. Are any two of following signs \& symptoms of infection both present and new to the patient? Note: laboratory values may have been obtained for inpatients but may not be available for outpatients.

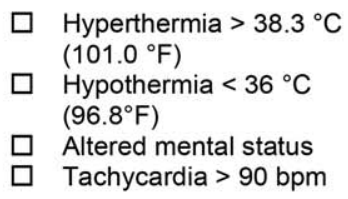

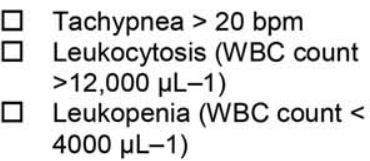

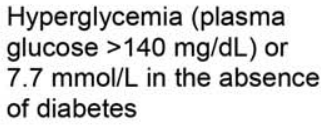
glucose $>140 \mathrm{mg} / \mathrm{dL}$ ) or $7.7 \mathrm{mmol} / \mathrm{L}$ in the absence of diabetes

If the answer is yes, to both questions 1 and 2 , suspicion of infection is present:

$\checkmark$ Obtain: lactic acid, blood cultures, CBC with differential, basic chemistry labs, bilirubin.

$\checkmark$ At the physician's discretion obtain: UA, chest $x$-ray, amylase, lipase, ABG, CRP, CT scan.

3. Are any of the following organ dysfunction criteria present at a site remote from the site of the infection that are NOT considered to be chronic conditions? Note: in the case of bilateral pulmonary infiltrates the remote site stipulation is waived.

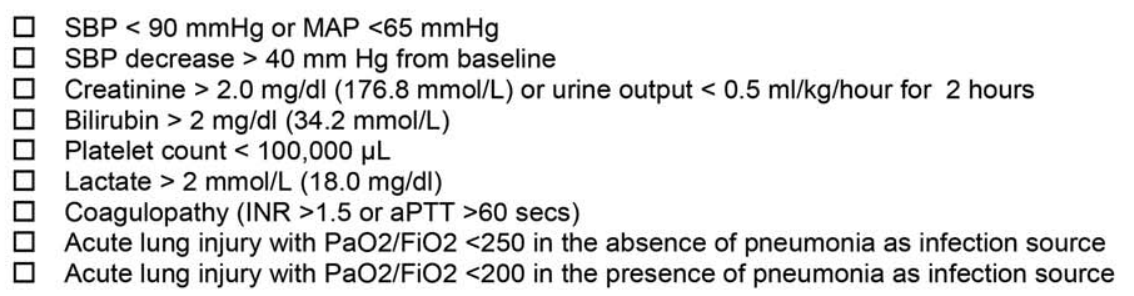

If suspicion of infection is present AND organ dysfunction is present, the patient meets the criteria for SEVERE SEPSIS and should be entered into the severe sepsis protocol.

Date: (circle: $\mathrm{dd} / \mathrm{mm} / \mathrm{yy}$ or $\mathrm{mm} / \mathrm{dd} / \mathrm{yy}$ )

Time: (24 hr. clock)

Version 7.2.13

FIG. 1. Evaluation for severe sepsis screening tool. This checklist is designed to prompt the nurse to screen every patient during every shift for new signs of sepsis and organ dysfunction (Checklist is available at: http://www.survivingsepsis.org/SiteCollectionDocuments/ScreeningTool.pdf).

and nurse leaders/champions. We found that engaging local clinical leaders in the early recognition and management of a severe sepsis QI program is imperative for the program's success. Hospitalists are often the physician leaders for the inpatient wards, so it is essential to secure their early engagement, support, and leadership. Moreover, though collaboration with ED and ICU physicians may be useful, as described 


\begin{tabular}{ll}
\hline TABLE 3. & Critical Steps Prior to Initiating a Ward \\
Sepsis-Detection Program \\
\hline 1. & Obtain administrative support (ie, funding for data collection, project lead, informatics) \\
2. & Align with ED and ICU \\
3. & Identify 1 ward to pilot the program \\
4. & Establish unit-based champions on each shift (nurse, physician) \\
5. & Review ward workflow \\
6. & Develop nurse screening tool \\
7. & Provide education \\
\hline
\end{tabular}

NOTE: Abbreviations: ED, emergency department; ICU, intensive care unit.

above, a hospitalist champion is likely to be more effective at educating other hospitalists about the program, overcoming physician resistance, and facilitating change.

Depending on a hospital's size and workflows, designated ward- or shift-based hospitalists and nurses as champions can serve as key resources to support implementation. These individuals help establish mutual respect and a common mental model of how sepsis can evolve in ward patients. Even more important, by providing assistance with both the screening tool as well as with recognition itself, these individuals not only speed implementation, but also protect against rough patches (ie, those instances where workflow changes run into resistance).

\section{EDUCATION}

Diagnosing sepsis is not always easy, making education on sepsis recognition, evaluation, and treatment necessary prior to implementation. Retention of knowledge over time through review and refresher courses are methods we used in the program. Providing background material explaining why education is necessary and providing physicians and nurses with materials to help them recall the information over time were developed at several sites. Resources included sepsis posters, identification-size badge cards with the sepsis bundle elements, and bulletin boards on the wards with information to reinforce sepsis recognition, evaluation, and treatment. Education for the ward-centric program included an overview of the SSC guidelines, supportive literature, sepsis definitions, description of the infection's systemic manifestations, criteria for identification of new- onset organ dysfunction, and the details on current severe sepsis 3- and 6-hour bundle requirements. We made clinicians aware of resources available on the SSC website. ${ }^{12}$ Data emphasizing the incidence of sepsis, as well as outcomes and motives for the QI wards program, were incorporated during the collaborative meetings. Data can serve as strong motivators for action (eg, highlighting current incidence rates). Many hospitals combined presentation of these aggregate data with local review of selected cases of severe sepsis that occurred in their own wards.

Understanding that the training for and experiences of ED, ICU, and ward nurses varies, nurse education contained critical assessment skills in determining when to suspect a new or worsening infection. Training nurses to complete a comprehensive daily infection assessment may help them overcome uncertainty in judgement. Assessment skills include examination of invasive lines, surgical sites, wounds, and presence of a productive cough. Equally important, patients being treated for an infection would benefit from a daily assessment for improvement or worsening of the infection. Information uncovered may identify early signs of organ failure in addition to infections that may need further evaluation and treatment. Education provides knowledge, but achieving program success relies heavily on staff accepting that they can make a difference in sepsis patient identification, management, and outcomes.

\section{SCREENING METHODS, COMMUNICATION, AND PROTOCOLS}

The SSC tool for severe sepsis facilitates screening for (1) confirmed or suspected infection, (2) presence of 2 or more systemic manifestations of infection, and (3) acute organ dysfunction. This tool was the basis for the "do" (screening) portion of the PDSA model.

\section{Continuous Screening}

Technology can facilitate early recognition of severe sepsis with EHR-based surveillance screening tools. Surveillance may include continuous review of vital signs and laboratory values with an automated alerting system. A valuable feature of the screening tool alert is the incorporation of the nurse's assessment. Decision support can improve the process by providing advice with systems requiring a reason to override the advice. ${ }^{13}$ For example, an alert may include input from the nurse to determine if the abnormal data are thought to be related to an infectious process or due to another cause. If a suspected or confirmed infection is identified, further surveillance screening can include review of blood pressure readings and laboratory data to determine if organ dysfunction is present. If organ dysfunction criteria are identified, the alert can prompt the nurse to notify the physician to discuss whether the organ dysfunction is new and related to the infection and if implementation of the severe sepsis bundles is indicated (Figure 2). Additional continuous screening models may include variations of the example provided to include alerts to other clinicians or a response team.

An automated screening tool within the EHR can be useful because the system continuously scans to identify signs and symptoms of sepsis, thus providing screening consistency, and offers data on the back end to be used as a mechanism for feedback to monitor effectiveness. Challenges with EHR severe sepsis alert development are resource allocation, testing, education, and ongoing evaluation and feedback. Other challenges include the potential for alert fatigue (false positive) and inappropriate response (false negative) to the infection prompt, thereby 
Severe Sepsis Alert with SBAR Embedded

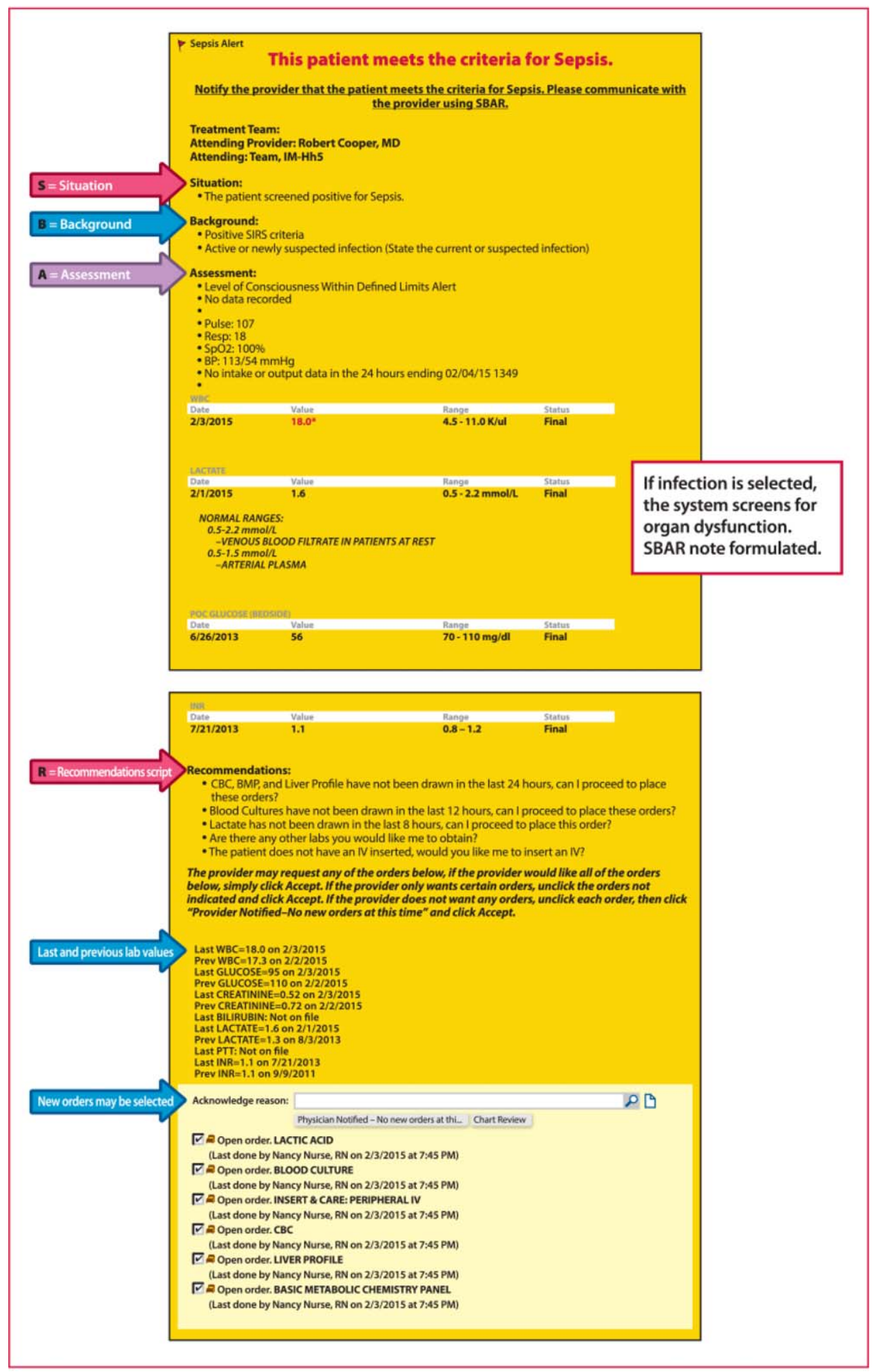

Q 2015 Epic Systems Corporation. Used with permission.

FIG. 2. Severe sepsis alert with situation, background, assessment, recommendation (SBAR) embedded. Abbreviations: BMP, basic metabolic panel; BP, blood pressure; CBC, complete blood count; INR, International Normalized Ratio; IV, intravenous; PTT, partial thromboplastin time; SIRS, systemic inflammatory response syndrome; SpO2, saturation of peripheral oxygen; WBC, white blood cells.

halting the next step in automated screening for organ dysfunction. Time to complete an automated screening tool varies based on strategic design and user understanding.

\section{Screening Checklist}

Whereas EHR tools may be effective in early recognition of sepsis, not all sites will have the capability to use these tools because of lack of informatics support, 


\title{
Example of ineffective communication of severe sepsis:
}

\author{
Nurse: Hello, Dr. Smith. Your patient in 1055 window has a fever and is not feeling \\ well. Would you like to order something for the fever? \\ Physician: Place an order for acetaminophen 500 mg every 4-6 hours as needed. I
}

will be in to see the patient after I finish outpatient office hours.

Application of SBAR for effective communication of severe sepsis:

Nurse: Hello, Dr. Smith. This is Mary Jones; I am the primary nurse caring for James Woods in $1055 \mathrm{~W}$.

Situation: Mr. Woods was admitted 1/1/2015 with cellulitis of his left lower extremity. He states that the pain in his leg has increased and the redness has extended since admission.

Background: Mr. Woods is a 78-year-old man with a history of hypertension and diabetes. He has reported a wound on his left lower leg present for more than two weeks. He arrived in the emergency department last night reporting increased pain, redness, drainage, and foul smelling odor.

Assessment: Mr. Woods' vital signs this morning revealed a temperature of 101.5, heart rate of 105, and respiratory rate of 22, and his current blood pressure is 95/40. His most recent laboratory values are from 1/1/15 at $2 \mathrm{pm}$. His WBCs were 12.2. He had blood cultures completed in the emergency department and the results are pending. He has not had any laboratory orders since admission to the emergency department.

Recommendation: I would like to order a chemistry panel, $C B C$ with differential, and lactic acid.

Physician: Repeat and confirm the information provided by Nurse Jones. The physician may agree with the recommendations and/or place the orders or may determine that the patient be monitored in a higher level of care.

FIG. 3. Script for communicating severe sepsis. Abbreviations: $C B C=$ complete blood count; WBC, white blood cells.

cost of development, and absence of an EHR in some hospitals. ${ }^{14}$ An alternative to continuous screening is a sepsis checklist such as the severe sepsis screening tool (Figure 1). The checklist is designed to prompt nurses to screen every patient during every shift for new signs of sepsis and organ dysfunction.

The checklist ensures that 3 key issues are considered: presence of a suspected or confirmed infection, systemic manifestations of inflammation, and physiological manifestations of organ dysfunction. The paper tool is simple to use and can be completed in 10 to 20 minutes. It requires the nurse to review the progress notes, vital signs, and laboratory test results. Although the time investment seems onerous, the gain in consistency of screening and treatment compensates for the extra effort. Review of the checklist also provides a locus for feedback and new improvement cycles.

\section{Scripted Communication}

Once a patient with severe sepsis is identified, communicating this finding to the rest of the clinical team is essential. Because communication skills are not always emphasized in QI projects, we decided to emphasize a structured approach. We provided clinicians with scripts based on the SBAR (situation, background, assessment, and recommendation) technique aimed to improve communication (Figure 3). ${ }^{15,16}$ Using the SBAR technique also supports our efforts to build nurses' confidence and willingness to employ protocols that give them greater autonomy.

\section{Nurse-Directed Protocols}

Skillful identification and management of severe sepsis patients constitute the foundation for implementation of nurse-directed protocols in this patient population. 
Such protocols promote autonomy and staff ownership. Severe sepsis protocols may include increasing the frequency of vital signs, placement of laboratory orders and, in sites with an established culture of increased nurse autonomy, initiation of intravenous access and a fluid bolus when specific criteria are met. Because nursing scope of practice varies from state to state and among hospitals, nurse-directed severe sepsis protocols generally require review of current site practice guidelines, physician agreement, and approval by the medical executive committee prior to implementation. Despite these differences, maximizing nurse leadership involvement and nurse autonomy can help propel the program forward. Protocols may be implemented based on knowledge level and resources on a particular ward. A workflow evaluation may be included in this process to define staff performing each step, what is being reported, and where and when data are recorded.

\section{DATA COLLECTION AND FEEDBACK}

Nurse screening drives the ward program and ensuring its consistency is the key to early patient identification. We made ongoing repeated evaluation of the appropriate use of the screening tool, time to physician notification, and time to follow-up intervention, a critical part of the study phase of the PDSA cycle. Once the nursing staff is consistently accurate and compliant (>90\%) with screening, random (eg, once per week) screening tool review may be more suitable, thus requiring fewer resources (see Supporting Information, Appendix 1, in the online version of this article).

\section{Data Collection}

A key to improvement is to study the process, which requires data collection to assess compliance. In our experience, timely clinician feedback, along with data, led to effective process change. Real-time data collection and discussion with the clinical team may lead to early recognition or intervention.

In our collaborative experience, we observed varied resources and timing for data collection across hospitals. For example, several participating sites had sepsis coordinators to collect data, whereas others relied on the quality department or nursing staff to collect data. Data may be collected concurrently (within 24 hours of severe sepsis presentation) or retrospectively. Retrospective data collection may allow for staff flexibility in data collection, but limits feedback to the clinicians. For example, with retrospective review, early recognition and treatment failure may go unrecognized until the data are analyzed and reported, which can be months after the patient has been discharged or expired.

\section{Feedback to Caregivers}

A consistent feedback process, which can occur at the individual or group level, may lead to prompt improvement in severe sepsis management. An example of individual feedback would be providing the nurse with the elapsed time from antibiotic order to time of administration. Early in the implementation phase, frequent (daily or weekly) feedback is helpful to build team cohesiveness. An example of feedback to build the team may include a unit-based report on the last 5 severe sepsis patients managed by the group. Providing overall bundle compliance and outcome reports on a weekly and monthly basis will allow the clinical team to track progress. Examples of report cards and a dashboard are provided in the supplemental material, which highlight compliance with the bundle elements as well as time to achieve the bundle elements. (see Supporting Information, Appendix 2 and Appendix 3, in the online version of this article). Resources to evaluate and provide consistent data may require up to 10 to 15 hours per week for 1 unit. Automated reports may decrease the resources needed in collating and reporting data.

\section{OUTCOME MEASURES}

Although certainly important, mortality is not the only outcome measure worthy of measurement. Other relevant outcomes include transfers to a higher level of care and need for major supportive therapies (eg, dialysis, mechanical ventilation, vasopressor infusion). Whereas it is valuable to review transfers to a higher level of care, we emphasized that these are not necessarily adverse outcomes; in fact, in many cases such transfers are highly desirable. It is also important to track the overall impact of sepsis on hospital length of stay.

\section{SUMMARY/CONCLUSIONS}

Grounded in the Institute for Healthcare Improvement's PDSA QI model, we developed a program aimed at improving outcomes for severe sepsis ward patients. Our program's cornerstone is nurse-led checklist-based screening. Our faculty led learning sessions that concentrated on using a collaborative approach whose key components were education in early sepsis identification, use of a sepsis screening tool, and the SBAR method for effective communication. Pitfalls identified during the program included lack of knowledge for both nurses and physicians in early severe sepsis identification, resistance to routine screening, and lack of data collection and leadership support. The most successful participating sites were those with senior leadership backing, staff engagement, informatics support, and data collection resources. Ultimately, replicating a program such as ours will depend on team cohesiveness, and nurse empowerment through the use of nursedriven protocols. Programs like this may lead to progression toward standardizing practice (eg, antibiotic administration, fluid resuscitation), matching patient needs to resources, and building stronger partnerships between hospitalists and nurses.

Disclosures: This work was supported by a grant provided to the Society of Critical Care Medicine by the Gordon and Betty Moore Foundation (Early Identification and Management of Sepsis on the Wards). The work was supported by a grant from the Adventist Hospital System. As 
part of our agreement with the Gordon and Betty Moore Foundation, we made a commitment to disseminate our findings in articles such as this one. However, the Moore Foundation played no role in how we actually structured our articles, nor did they review or preapprove any of the manuscripts submitted as part of the dissemination component; the same was the case with the other sponsors. The authors report no conflicts of interest.

\section{References}

1. Levy MM, Dellinger RP, Townsend SR, et al. The Surviving Sepsis Campaign: results of an international guideline-based performance improvement program targeting severe sepsis. Intensive Care Med. 2010;36(2):222-231.

2. Levy MM, Dellinger RP, Townsend SR, et al. The Surviving Sepsis Campaign: results of an international guideline-based performance improvement program targeting severe sepsis. Crit Care Med. 2010; 38(2):367-374.

3. Levy MM, Rhodes A, Phillips GS, et al. Surviving Sepsis Campaign: association between performance metrics and outcomes in a 7.5 -year study. Intensive Care Med. 2014;40(11):1623-1633.

4. Rohde JM, Odden AJ, Bonham C, et al. The epidemiology of acute organ system dysfunction from severe sepsis outside of the intensive care unit. J Hosp Med. 2013;8(5):243-247.

5. Yealy DM, Huang DT, Delaney A, et al. Recognizing and managing sepsis: what needs to be done? BMC Med. 2015;13:98.

6. Sopena N, Heras E, Casas I, et al. Risk factors for hospital-acquired pneumonia outside the intensive care unit: a case-control study. Am J Infect Control. 2014;42(1):38-42.
7. Dellinger RP, Levy MM, Rhodes A, et al. Surviving Sepsis Campaign: international guidelines for management of severe sepsis and septic shock, 2012. Crit Care Med. 2013;41(2):580-637.

8. Nadeem E, Olin SS, Hill LC, Hoagwood KE, Horwitz SM. Understanding the components of quality improvement collaboratives: a systematic literature review. Milbank Q. 2013;91(2):354-394.

9. Langley GL, Moen R, Nolan KM, Nolan TW, Norman CL, Provost LP. The Improvement Guide: A Practical Approach to Enhancing Organizational Performance. 2nd ed. San Francisco, CA: Jossey-Bass; 2009.

10. Nembhard IM. Learning and improving in quality improvement collaboratives: which collaborative features do participants value most? Health Serv Res. 2009;44(2 pt 1):359-378.

11. Pronovost PJ, Weast B, Bishop K, et al. Senior executive adopt-a-work unit: a model for safety improvement. Jt Comm J Qual Saf. 2004; 30(2):59-68.

12. Surviving Sepsis Campaign. Available at: http://survivingsepsis.org/ Resources/Pages/default.aspx. Accessed September 24, 2015.

13. Roshanov PS, Fernandes N, Wilczynski JM, et al. Features of effective computerised clinical decision support systems: meta-regression of 162 randomised trials. BMJ. 2013;346:f657.

14. Bhounsule P, Peterson AM. characteristics of hospitals associated with complete and partial implementation of electronic health records. Perspect Health Inf Manag. 2016;13:1c.

15. Institute for Healthcare Improvement. SBAR technique for communication: a situational briefing model. Available at: http://www.ihi.org/ resources/pages/tools/sbartechniqueforcommunicationasituationalbriefingmodel.aspx. Accessed September 12, 2015.

16. Compton J, Copeland K, Flanders S, et al. Implementing SBAR across a large multihospital health system. It Comm J Qual Patient Saf. 2012;38(6):261-268. 